\title{
A new sample of large angular size radio galaxies
}

\section{The optical data}

\author{
L. Lara $^{1, \star}$, I. Márquez ${ }^{1, \star}$, W. D. Cotton ${ }^{2}$, L. Feretti ${ }^{3}$, G. Giovannini ${ }^{3,4}$, J. M. Marcaide ${ }^{5}$, and T. Venturi ${ }^{3}$ \\ 1 Instituto de Astrofísica de Andalucía (CSIC), Apdo. 3004, 18080 Granada, Spain \\ 2 National Radio Astronomy Observatory, 520 Edgemont Road, Charlottesville, VA 22903-2475, USA \\ 3 Istituto di Radioastronomia (CNR), via P. Gobetti 101, 40129 Bologna, Italy \\ 4 Dipartamento di Fisica, Universitá di Bologna, via B. Pichat 6/2, 40127 Bologna, Italy \\ 5 Departimento de Astronomía, Universitat de València, 46100 Burjassot, Spain
}

Received 3 August 2001 / Accepted 11 September 2001

\begin{abstract}
We constructed and presented in the first paper of this series a new sample of 84 large angular size radio galaxies by selecting from the NRAO VLA Sky Survey objects with angular size $\geq 4^{\prime}$, declination above $+60^{\circ}$ and total flux density at $1.4 \mathrm{GHz} \geq 100 \mathrm{mJy}$. In this paper we present optical spectra and images of those galaxies associated with the radio emission for which no redshift was known prior to our observations. Optical counterparts have been identified for all (but one) members of the sample. After our observations, a reliable spectroscopic redshift is available for 67 objects (80\%) from the sample. This paper, the second of a series of three, contributes to increase the number of well-defined samples of radio galaxies with ample spectroscopic information.
\end{abstract}

Key words. galaxies: active - galaxies: nuclei - galaxies: distances and redshift - radio continuum: galaxies

\section{Introduction}

Lara et al. (2001), hereafter Paper I, presented a new sample of large angular size radio galaxies selected from the NRAO $^{1}$ VLA $^{2}$ Sky Survey (NVSS; Condon et al. 1998). The sample, covering a sky area of $\pi$ steradians, was constructed under the following selection criteria: declination above $+60^{\circ}$, total flux density at $1.4 \mathrm{GHz}$ greater than $100 \mathrm{mJy}$ and angular size larger than $4^{\prime} .122$ radio sources were pre-selected and observed with the VLA with higher angular resolution at 1.4 and $4.9 \mathrm{GHz}$ for confirmation, and a total of 84 radio galaxies were selected for the final sample (see Table 2 in Paper I). In Paper I we have shown that, within the selection criteria, our sample is homogeneous and suitable for statistical studies. Moreover, our sample represents a substantial increase in the number of known giant radio galaxies (GRGs; defined as those

Send offprint requests to: L. Lara,

e-mail: lucas@iaa.csic.es

* Visiting Astronomer, German-Spanish Astronomical Center, Calar Alto, operated by the Max-Planck-Institut für Astronomie jointly with the Spanish National Commission for Astronomy.

1 National Radio Astronomy Observatory.

2 Very Large Array, operated by the NRAO. with a projected linear size ${ }^{3} \geq 1 \mathrm{Mpc}$ ), with 22 new objects belonging to this class. This sample, together with other new samples currently being studied by other groups (Schoenmakers et al. 2000, 2001; Machalski et al. 2001) raises the number of known GRGs to above one hundred.

The identification of the optical counterpart of the radio core emission, and the determination of its redshift, is a necessary step for the study of any sample of radio galaxies. In our case, many of the members of the sample were poorly known, both at radio and optical wavelengths. First, a reliable optical identification of the radio galaxies had not been possible previous to our work since only low angular resolution radio data existed and, consequently, only a poor determination of the radio core position was possible. The radio maps of the sample members presented in Paper I allowed us to obtain accurate positions of the core components (see Table 2) and, in most cases, to attempt successful identification of the associated galaxy on the Digital Sky Survey (DSS). Second, only 35\% of the galaxies in the sample had measured redshifts. Thus, it was necessary to tackle the task of measuring the redshift of all the remaining galaxies in order to obtain useful physical parameters for a subsequent study.

\footnotetext{
${ }^{3}$ We assume that $H_{0}=50 \mathrm{~km} \mathrm{~s}^{-1} \mathrm{Mpc}^{-1}$ and $q_{0}=0.5$.
} 
We present here (Paper II) images obtained with the $2.2 \mathrm{~m}$ telescope in Calar Alto of the optical counterparts of the sample galaxies for which no redshift was available in the literature at the time of the observations. We have made long-slit spectroscopy of these objects and determined the redshift for 46 (6 uncertain) of them. In the forthcoming third paper of this series, we will present a global analysis of the sample properties.

\section{Optical imaging and spectroscopy}

We made optical observations of 57 radio galaxies from the sample, pointing the $2.2 \mathrm{~m}$ telescope in Calar Alto (Spain) to the position of the radio core component. The log of the observing runs is given in Table 1 . We used the Calar Alto Faint Object Spectrograph (CAFOS) equipped with a SITe-1d $2048 \times 2048$ CCD. CAFOS allows direct imaging and spectroscopy, with a spatial scale of 0.53 per pixel with this CCD. To obtain the spectra, we used a medium resolution grism (200 $\mathrm{A} / \mathrm{mm})$, sensitive to the wavelength range of 4000 to $8500 \AA$, that provides a spectral resolution of $4.47 \AA /$ pixel.

The standard procedure followed during the observations was to take first one $300 \mathrm{~s}$ exposure image in the Johnson $R$-band in order to identify the optical host of the radio galaxy core component, whose position had already been accurately determined from VLA radio observations (Paper I) and registered on the DSS plates. Once the galaxy coincident with the radio core was located in the image, we obtained a spectrum with the CAFOS long-slit configuration, taking two equal exposures (usually $900 \mathrm{~s}$, or $1200 \mathrm{~s}$ for weaker objects) to be added in order to reject the cosmic rays. We used a $2^{\prime \prime}$ wide slit placed northsouth, except on J1220+636, J1251+756, J0819+756 and $\mathrm{J} 1251+787$, for which a $-45^{\circ}$ slit rotation was applied in order to avoid contamination from nearby bright stars. Some objects required longer exposures in the $R$-band in order to detect the associated optical galaxy, and in some of the weaker galaxies, although detected, we did not attempt to obtain a spectrum.

The data reduction and calibration were performed following standard procedures with the $\mathrm{IRAF}^{4}$ software, involving dark and flat field corrections. Wavelength calibration was carried out using exposures of mercuryhelium-rubidium arc lamps taken just before or after the target exposure. No flux calibration of the spectra was attempted since the atmospheric conditions were far from photometric during most of the observing nights. In fact, some of the resulting spectra have quite a low signal to noise ratio due to bad weather conditions (e.g. J0757+826 or J0828+632). Examining the intensity profile along the slit on each spectrum, we determined the most adequate

${ }^{4}$ IRAF is the Image Reduction and Analysis Facility made available to the astronomical community by the National Optical Astronomy Observatories, which are operated by the Association of Universities for Research in Astronomy (AURA), Inc., under contract with the U.S. National Science Foundation.
Table 1. Optical observations.

\begin{tabular}{lccc}
\hline Nights & $\begin{array}{c}\text { Average seeing } \\
\left({ }^{\prime \prime}\right)\end{array}$ & $\sigma$ & Code $^{a}$ \\
\hline Sep. 26th 1997 & 1.88 & 0.36 & a1 \\
Oct. 30th 1998 & 1.98 & 0.33 & b1 \\
Oct. 31st 1998 & 1.16 & 0.14 & b2 \\
Nov. 1st 1998 & 1.23 & 0.17 & b3 \\
Nov. 2nd 1998 & 1.72 & 0.22 & b4 \\
Dec. 8th 1998 & 1.08 & 0.17 & c1 \\
Dec. 9th 1998 & 1.02 & 0.14 & c2 \\
\hline
\end{tabular}

${ }^{a}$ Code used in Table 2 to indicate the epoch of observation of each source.

region for the determination and subtraction of the sky contribution, and the region centered on the target galaxy which we summed in order to create the corresponding 1dimension spectrum.

Images of the optical counterpart of the observed radio galaxies and their spectra are shown in Fig. 1. The average seeing $(F W H M)$ determined for each night using foreground stars in the different $R$-band images is displayed in Table 1 , and varies from $2^{\prime \prime}$ on the night of 30th October 1998 to $1^{\prime \prime}$ on 9 th December 1998. Two prominent atmospheric absorption bands at $\lambda=7604 \AA$ and $\lambda=6882 \AA$, corresponding to the Fraunhofer $A$ and $B$ bands of molecular oxygen, are marked with vertical lines on the spectra in Fig. 1.

\section{Notes on singular sources}

In this section we make a brief description of those observed galaxies which show some type of remarkable properties in the optical regime (luminosity, spectrum or nearby environment), when compared with the rest of the sample:

J0342+636: A spheroidal galaxy with a prominent broad $\mathrm{H} \alpha$ emission line. A weaker broad $\mathrm{H} \beta$ line and [OIII] narrow emission lines are also detected. J0342+636 presents a close companion at an angular distance of $6.8^{\prime \prime}$ in position angle $(\mathrm{PA})$ of $-53^{\circ}$, which corresponds to a linear projected distance of $20.5 \mathrm{kpc}$ (if both galaxies are assumed to be at the same redshift).

J0455+603: At the time of the optical observations we had not correctly identified the core component in the radio structure, and consequently did not find the associated galaxy after two exposures of $300 \mathrm{~s}$ each. The core component of J0455+603 was misidentified with a bright and compact feature in our $1.4 \mathrm{GHz}$ VLA map (see Paper I). However, we have now identified the core with a weaker component with flat spectrum at $16^{\prime \prime}$ in NW direction from the previously assumed core, which is coincident with the galaxy marked in Fig. 1. Coordinates in Table 2 have been corrected with respect to those listed in Paper I.

J0502+670: The host galaxy of this Fanaroff-Riley type I radio source (FR I; Fanaroff \& Riley 1974) is a bright elliptical at $z=0.085$ with an spectrum characterized by the presence of stellar absorption features. It has 

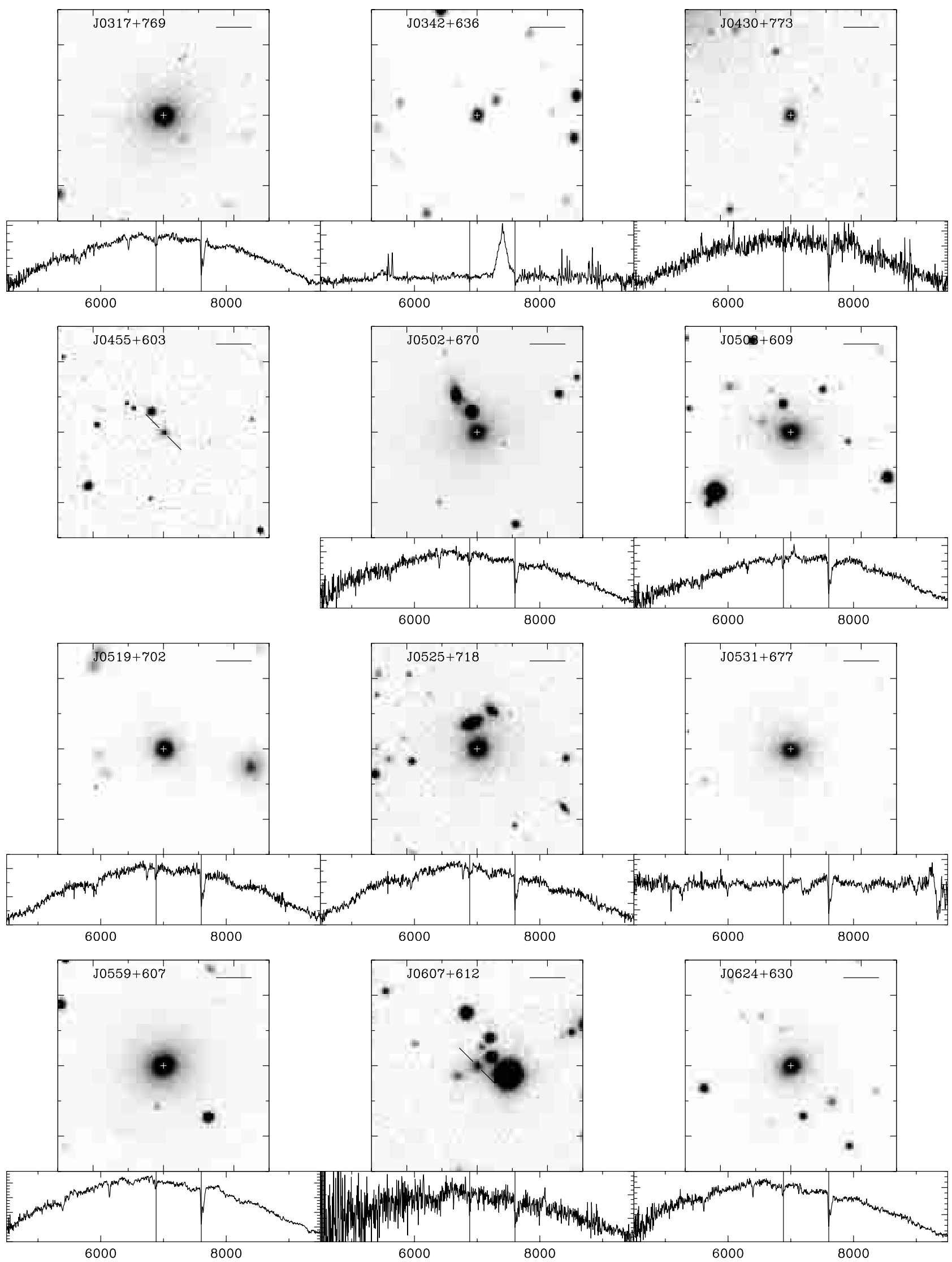

Fig. 1. Optical images of the galaxies identified with the radio sources of the sample. The white cross (or broken line) helps to identify the galaxy coincident with the position of the radio core. The segment on upper right corresponds to an angular size of $10^{\prime \prime}$. Below each image we display the optical spectrum, obtained with a long slit of $2^{\prime \prime}$ width. Flux units are arbitrary and wavelengths are given in $\AA$. The two vertical solid lines mark the position of atmospheric absorption bands produced by $\mathrm{O}_{2}$. 
L. Lara et al.: A new sample of large angular size radio galaxies. II.
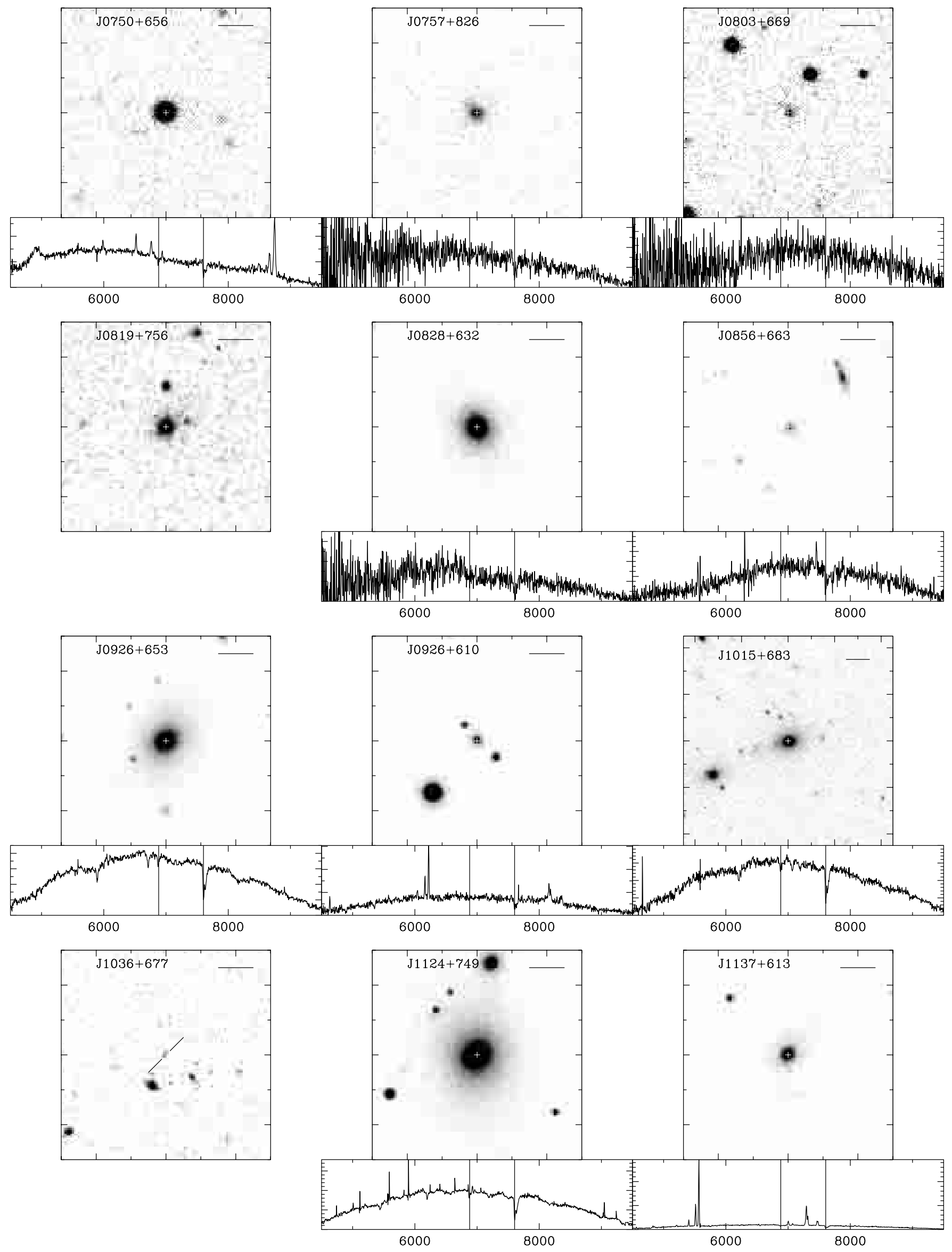

Fig. 1. continued. 

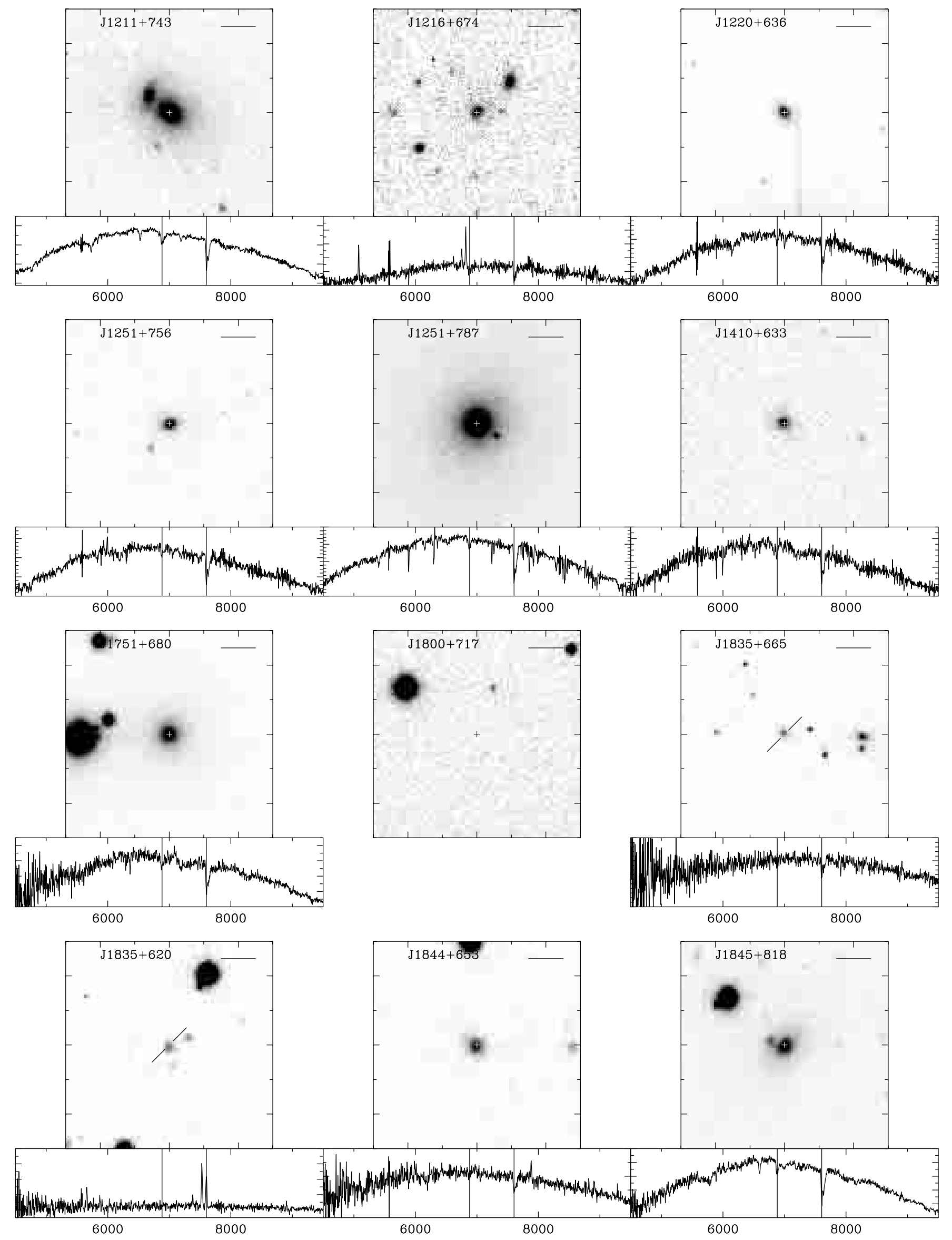

Fig. 1. continued. 
L. Lara et al.: A new sample of large angular size radio galaxies. II.
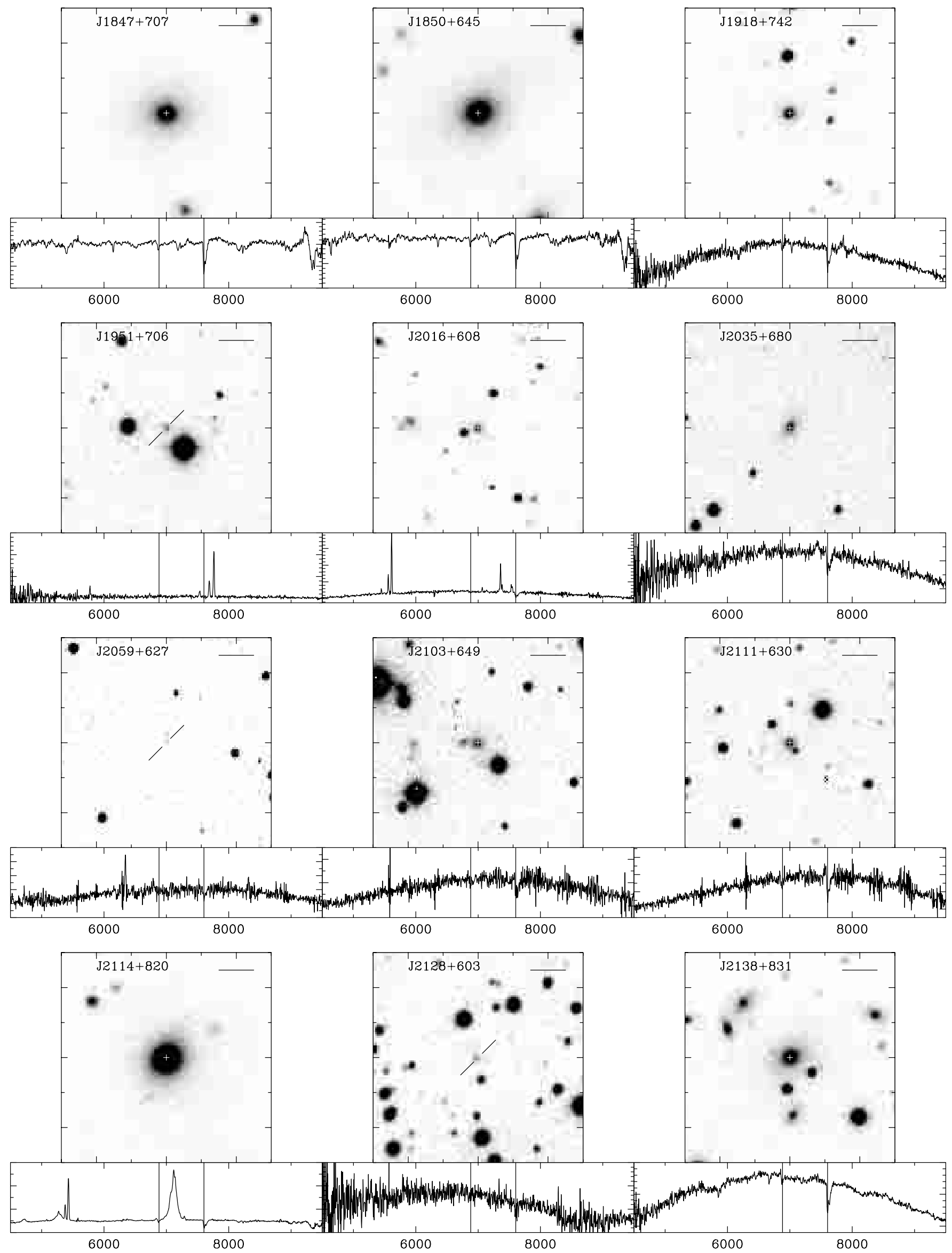

Fig. 1. continued. 

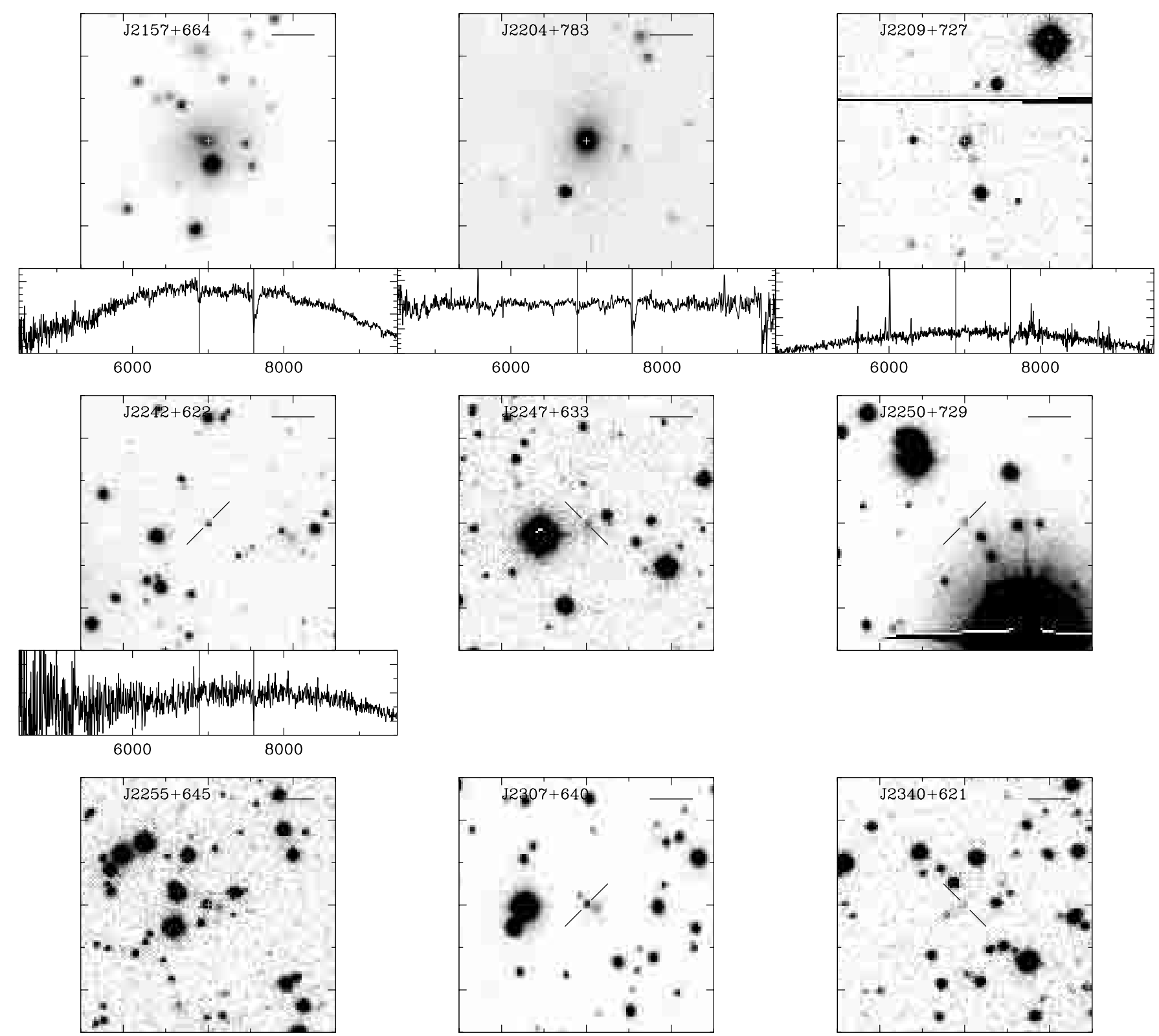

Fig. 1. continued.

a close companion separated by $12^{\prime \prime}$ at PA of $29^{\circ}$, which corresponds to $25.7 \mathrm{kpc}$ if assumed to be at the same redshift. There is a foreground star $\left(m_{R} \sim 13\right)$ between the two galaxies.

J0525+718: A bright elliptical galaxy is the source of this low power radio galaxy. There are two nearby galaxies at $7.6^{\prime \prime}$ in $\mathrm{PA}+2^{\circ}$ and at $12^{\prime \prime}$ in $\mathrm{PA}-21^{\circ}$, which if assumed to be at the same redshift of $\mathrm{J} 0525+718$ correspond to projected distances of 26 and $41 \mathrm{kpc}$, respectively.

J0750+656: The most distant radio source in our sample, at $z=0.747$. It is optically identified with a 16.4 magnitude quasar type object.

$\mathbf{J 1 0 1 5}+\mathbf{6 8 3}$ : Two galaxies in the field can be identified with components in the complex radio structure of this source (see Fig. 2), which confirms that J1015+683 results from the superposition of two distinct radio galaxies

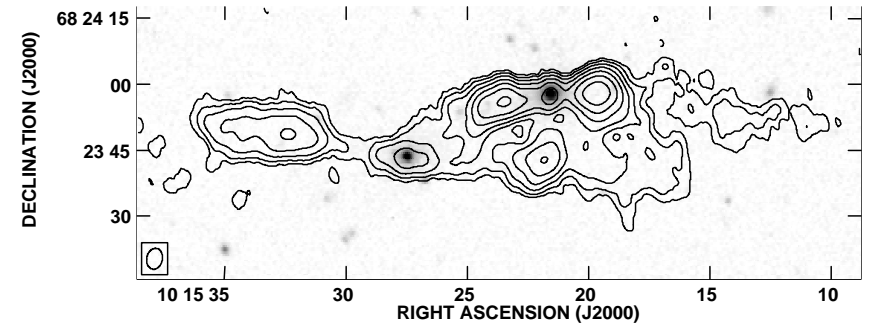

Fig. 2. Superposition of a radio image of $\mathrm{J} 1015+683$ at $4.9 \mathrm{GHz}$ (Paper I) with the Johnson $R$-band filter optical image of the same field.

with their main axes in a similar (projected) direction. The angular distance of the two galaxies is $35.4^{\prime \prime}$, which at the redshift of the northern one $(z=0.199)$ corresponds to $149 \mathrm{kpc}$. The classification of this radio galaxy as a giant 
(Paper I) must be postponed until a redshift determination of the southern galaxy.

$\mathbf{J 1 1 3 7 + 6 1 3 :}$ This radio galaxy, of Fanaroff-Riley type II (FR II), originates from an spheroidal galaxy. Its spectrum presents high signal to noise ratio emission lines $(\mathrm{H} \beta,[\mathrm{OIII}],[\mathrm{OI}], \mathrm{H} \alpha,[\mathrm{NII}],[\mathrm{SII}])$.

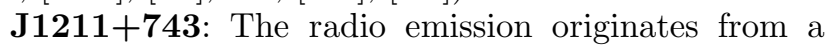
bright elliptical galaxy. It has a nearby companion at $7.5^{\prime \prime}$ in $\mathrm{PA}+51^{\circ}$, which corresponds to $19.5 \mathrm{kpc}$ if assumed to be at the same redshift of J1211+743. There are no emission features in the spectrum, which is dominated by the continuum and some stellar absorption lines.

$\mathbf{J 1 8 0 0 + 7 1 7}$ : This is the only object in our sample for which no hint of the optical counterpart of the radio emission is found. The cross in Fig. 1 represents the position of the radio core of this FR II radio galaxy. We made three $300 \mathrm{~s}$ exposures on the field centered at this position but nothing was detected. The $R$-band extinction is only of 0.1 magnitudes in this region (Schlegel et al. 1998), so the associated galaxy must either be very distant or intrinsically weak. A limit to its apparent magnitude $\left(m_{R}>19.5\right)$ can be estimated from the magnitude of a weak object located $13^{\prime \prime}$ northward from the radio core position.

$\mathbf{J 1 8 4 5 + 8 1 8}$ : It is identified with an elliptical galaxy. At a distance of $4.4^{\prime \prime}$ in $\mathrm{PA}+76^{\circ}$ from the galaxy center, there is a small, possibly elongated feature. It is coincident with the direction of the radio jet, although if it is related to the jet is not clear. Most probably, it is a small galaxy close to the radio emitting elliptical galaxy, at a projected distance of $12.5 \mathrm{kpc}$ if assumed to be at the same redshift. The galaxy spectrum presents only stellar absorption lines.

J2059+627: We obtained only a bad quality optical image of this field, although sufficient to identify a very weak feature associated with the radio core. Two $1200 \mathrm{~s}$ long-slit exposures revealed a weak continuum with prominent [OIII] emission lines.

J2114+820: A peculiar low power radio galaxy of FR I type associated with an elliptical galaxy which shows prominent broad $\mathrm{H} \alpha, \mathrm{H} \beta$ and $\mathrm{H} \gamma$ emission lines, and narrow [OIII] lines (Lara et al. 1999). The galaxy has a bright compact core associated to the nuclear activity.

$\mathbf{J 2 1 3 8 + 8 3 1 : ~ T h e ~ o r i g i n ~ o f ~ t h i s ~ r a d i o ~ s o u r c e ~ i s ~ a ~ b r i g h t ~}$ elliptical galaxy residing in the cluster Abell 2387. Its spectrum shows no emission lines, being characterized by stellar absorption lines.

$\mathbf{J 2 1 5 7 + 6 6 4 :}$ : We made three $300 \mathrm{~s}$ exposures on this galaxy which revealed an extended elliptical galaxy with an apparent double core in east-west direction. The radio core is coincident with the western feature. No emission lines are found in the spectrum. The galaxy, with a galactic latitude of $+9.2^{\circ}$, lies in a crowded field, with possibly smaller galaxies around, and a bright foreground star located nearby southwards.

J2247+633: We made two 300 s exposures on the field centered at the radio core position, and found a very weak feature coincident with the radio core. The galactic latitude of this galaxy is only of $+3.7^{\circ}$. We did not attempt to obtain the spectrum of this galaxy.

J2250+729: We made three 300 s exposures on the field centered at the radio galaxy core. A very weak feature associated with the radio core was found, close to a bright star with apparent $m_{R}=10.1$. The galactic latitude of this galaxy is $+12.1^{\circ}$. We did not attempt to obtain the spectrum of this galaxy.

J2340+621: We made two 300 s exposures and tentatively found the optical counterpart of this radio galaxy in a crowded star field. The galactic latitude is only $+0.4^{\circ}$ and the $R$-band extinction in this region is of 4.0 magnitudes (Schlegel et al. 1998). We did not attempt to obtain the optical spectrum of this galaxy.

\section{Results}

In Table 2 we present the main results derived from the observations reported in this paper:

Column 1: IAU source name at epoch J2000.

Columns 2, 3: right ascension and declination (J2000).

Column 4: galactic latitude.

Column 5: code defined in Table 1 indicating the epoch of observation.

Column 6: integration time for the spectroscopic observations.

Column 7: redshift derived from our observations or from the literature.

Column 8: notes about the optical spectrum: SEL strong emission lines present; WEL - weak emission lines; AbL - absorption lines only.

Column 9: apparent photographic magnitudes derived from the red band Palomar survey (DSS R-band). Magnitudes of faint galaxies (above the 16th magnitude) have been obtained from the USNO-A2.0 catalogue (Monet et al. 1998), and compared with the APM catalogue (http://www.ast.cam.ac.uk/ a apmcat). In general, we found good agreement between these two catalogues, but we adopted the mean value in the few cases where the discrepancy was larger than 0.4 magnitudes. When USNO-A2.0 did not provide information on a requested galaxy, we adopted the APM magnitude. The magnitudes of brighter galaxies (up to the 16th magnitude) have been determined directly from the DSS using the available photometric calibration (Doggett et al. 1996). However, we note that the apparent magnitudes of the brighter extended galaxies are subject of large errors (sometimes larger than 3 magnitudes) probably due to the non-linear behavior of the photographic plates.

Column 10: effective radius of the galaxies derived by fitting a $r^{1 / 4}$ profile to the brightness distribution. Although the low sensitivity or quality of some galaxy images prevented us from obtaining reasonable fits for them, in all possible cases the profile was consistent with that typical of elliptical galaxies.

Column 11: type of radio structure: I and II stand for FR I and FR II type radio galaxies, respectively. Distinction between the two classes is based solely on morphological 
Table 2. Sample of large angular size radio galaxies from the NVSS survey.

\begin{tabular}{|c|c|c|c|c|c|c|c|c|c|c|}
\hline Name & $\begin{array}{c}\text { RA (J2000.0) } \\
\left(\begin{array}{lll}(\mathrm{h} & \mathrm{m} & \mathrm{s}\end{array}\right)\end{array}$ & 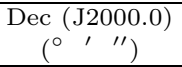 & $\begin{array}{c}b \\
\left({ }^{\circ}\right)\end{array}$ & Epoch & $\begin{array}{r}T_{\exp } \\
(\mathrm{s})\end{array}$ & $z$ & Notes & $\begin{array}{c}m_{R} \\
(\mathrm{mag})\end{array}$ & $\begin{array}{c}r_{\mathrm{e}} \\
(\mathrm{kpc})\end{array}$ & $\begin{array}{l}\text { Radio } \\
\text { Type }\end{array}$ \\
\hline J0109+731 & 010944.265 & 731157.17 & 10.4 & & & $0.181^{a}$ & SEL & - & - & II \\
\hline J0153+712 & 015325.786 & $71 \quad 1506.53$ & 9.0 & & & $0.022^{a}$ & SEL & 10.4 & - & I \\
\hline $\mathrm{J} 0317+769$ & $03 \quad 1754.061$ & $7658 \quad 37.82$ & 16.5 & b1 & $2 \times 900$ & 0.094 & $\mathrm{AbL}$ & 14.7 & 37.0 & I \\
\hline J0318+684 & $\begin{array}{lll}03 & 18 & 19.026\end{array}$ & $\begin{array}{lll}68 & 29 & 32.08\end{array}$ & 9.4 & & & $0.090^{b}$ & WEL & 17.5 & - & II \\
\hline J0342+636 & $\begin{array}{lll}03 & 42 & 10.148\end{array}$ & 633933.73 & 6.8 & a1 & $2 \times 900$ & 0.128 & SEL & 17.9 & - & II \\
\hline $\mathrm{J} 0430+773$ & 043049.490 & 772258.44 & 19.5 & b1 & $2 \times 1200$ & 0.215 & WEL & 18.5 & 10.4 & II \\
\hline $\mathrm{J} 0455+603$ & 045545.847 & 602348.94 & 10.6 & $\mathrm{c} 1$ & & - & - & - & - & I \\
\hline $\mathrm{J} 0502+670$ & 050254.732 & $\begin{array}{lll}67 & 02 & 30.15\end{array}$ & 15.2 & b2 & $2 \times 1200$ & 0.085 & $\mathrm{AbL}$ & 13.9 & 28.9 & I \\
\hline J0508+609 & $0508 \quad 27.258$ & 605627.48 & 12.2 & b2 & $2 \times 900$ & 0.071 & WEL & 14.6 & 34.6 & I \\
\hline J0519+702 & $\begin{array}{lll}05 & 19 & 17.132\end{array}$ & 701348.68 & 18.1 & b1 & $2 \times 900$ & 0.144 & $\mathrm{AbL}$ & 16.1 & 33.1 & I \\
\hline $\mathrm{J} 0525+718$ & 052527.094 & $7152 \quad 39.25$ & 19.4 & b2 & $2 \times 900$ & 0.150 & $\mathrm{AbL}$ & 15.9 & 26.8 & I \\
\hline $\mathrm{J} 0531+677$ & 053125.925 & 674350.23 & 17.9 & a1 & $2 \times 900$ & 0.017 & $\mathrm{AbL}$ & 8.8 & 3.0 & I \\
\hline J0546+633 & 054624.622 & 632132.50 & 17.2 & & & $0.049^{a}$ & $? ?$ & 10.9 & - & I \\
\hline $\mathrm{J} 0559+607$ & 055938.690 & 604400.96 & 17.5 & b2 & $2 \times 900$ & 0.042 & $\mathrm{AbL}$ & 12.6 & 14.3 & I \\
\hline J0607+612 & 060734.919 & 611443.52 & 18.6 & b3 & $2 \times 1200$ & 0.227 & WEL & - & - & $\mathrm{I} / \mathrm{II}$ \\
\hline J0624+630 & 062429.063 & 630402.50 & 21.1 & b3 & $2 \times 900$ & 0.085 & $\mathrm{AbL}$ & 14.5 & 10.4 & I \\
\hline J0633+721 & 063340.842 & 720924.92 & 24.4 & & & $0.090^{b}$ & $\mathrm{AbL}$ & 15.0 & - & $\mathrm{I} / \mathrm{II}$ \\
\hline J0654+733 & 065426.525 & $\begin{array}{lll}73 & 19 & 50.36\end{array}$ & 26.1 & & & $0.115^{b}$ & SEL & 15.2 & - & II \\
\hline J0750+656 & 075034.425 & 654125.50 & 30.6 & b1 & $2 \times 900$ & 0.747 & SEL & 16.4 & - & II-QSS \\
\hline J0757+826 & 075735.172 & 823940.86 & 29.0 & b4 & $3 \times 1200$ & $0.087 ?(0.06)$ & $\mathrm{AbL}$ & 12.0 & 9.6 & I \\
\hline J0803+669 & 080345.829 & $\begin{array}{lll}66 & 56 & 11.39\end{array}$ & 31.9 & b3 & $2 \times 1200$ & $0.247 ?(0.37)$ & $\mathrm{AbL}$ & 19.3 & - & II \\
\hline $\mathrm{J} 0807+740$ & $0807 \quad 10.070$ & 740041.58 & 31.2 & & & $0.120^{b}$ & SEL & 15.8 & - & I \\
\hline J0819+756 & $08 \quad 1950.504$ & $75 \quad 38 \quad 39.53$ & 31.7 & $\mathrm{c} 2$ & $1 \times 900$ & $0.232^{b}$ & SEL & 17.7 & 24.8 & II \\
\hline J0825+693 & $08 \quad 2559.770$ & 692038.59 & 33.5 & & & $0.538^{a}$ & SEL & - & - & II \\
\hline J0828+632 & 082856.363 & 631345.05 & 34.9 & b4 & $2 \times 1200$ & $(0.09)$ & - & 13.7 & $(10.7)$ & I/II \\
\hline J0856+663 & $\begin{array}{lll}08 & 56 & 16.260\end{array}$ & 662126.50 & 37.1 & b3 & $2 \times 1200$ & 0.489 & WEL & 19.7 & - & II \\
\hline J0926+653 & 092600.822 & $\begin{array}{lll}65 & 19 & 22.88\end{array}$ & 40.3 & b2 & $2 \times 900$ & 0.140 & $\mathrm{AbL}$ & 15.0 & 42.3 & I \\
\hline J0926+610 & 092653.408 & 610024.87 & 42.0 & b2 & $1 \times 900$ & 0.243 & SEL & 17.2 & 25.8 & II \\
\hline $\mathrm{J} 0939+740$ & 093946.833 & $7405 \quad 30.78$ & 37.0 & & & $0.122^{b}$ & $\mathrm{AbL}$ & 14.8 & - & I \\
\hline J0949+732 & 094946.157 & 731423.82 & 38.1 & & & $0.058^{a}$ & SEL & 12.8 & - & II \\
\hline $\mathrm{J} 1015+683$ & 101521.620 & 682358.24 & 42.7 & $\mathrm{c} 1$ & $2 \times 900$ & 0.199 & $\mathrm{AbL}$ & 15.1 & 31.6 & $?$ \\
\hline $\mathrm{J} 1036+677$ & 103641.237 & $67 \quad 4753.44$ & 44.6 & $\mathrm{c} 1$ & & - & - & - & - & II \\
\hline $\mathrm{J} 1124+749$ & 112447.045 & $74 \quad 55 \quad 45.31$ & 40.9 & $\mathrm{c} 1$ & $1 \times 900$ & 0.052 & WEL & 10.7 & 34.9 & I \\
\hline $\mathrm{J} 1137+613$ & 113721.289 & $\begin{array}{lll}61 & 20 & 01.88\end{array}$ & 53.6 & $\mathrm{c} 1$ & $2 \times 900$ & 0.111 & SEL & 16.2 & 18.8 & II \\
\hline $\mathrm{J} 1211+743$ & 121158.710 & 741904.12 & 42.5 & $\mathrm{c} 1$ & $2 \times 900$ & 0.107 & $\mathrm{AbL}$ & 12.6 & 49.1 & I/II \\
\hline $\mathrm{J} 1216+674$ & $12 \quad 16 \quad 37.239$ & 672441.97 & 49.4 & $\mathrm{c} 2$ & $2 \times 900$ & 0.362 & SEL & 18.7 & 41.0 & II \\
\hline $\mathrm{J} 1220+636$ & $12 \quad 2036.477$ & 634143.82 & 53.1 & $\mathrm{c} 2$ & $2 \times 900$ & 0.188 & $\mathrm{AbL}$ & 15.7 & - & II \\
\hline $\mathrm{J} 1247+673$ & 124733.319 & $\begin{array}{lll}67 & 23 & 16.34\end{array}$ & 49.7 & & & $0.107^{a}$ & $\mathrm{AbL}$ & 14.7 & - & II \\
\hline $\mathrm{J} 1251+756$ & 125105.977 & 753738.94 & 41.5 & $\mathrm{c} 2$ & $2 \times 900$ & 0.197 & WEL & 16.4 & 6.9 & II \\
\hline $\mathrm{J} 1251+787$ & 125123.839 & 784236.29 & 38.4 & c2 & $900+700$ & 0.045 & $\mathrm{AbL}$ & 10.4 & 17.1 & I \\
\hline $\mathrm{J} 1313+696$ & 131358.878 & $6937 \quad 18.74$ & 47.4 & & & $0.106^{a}$ & SEL & 16.0 & - & II \\
\hline $\mathrm{J} 1410+633$ & 141030.609 & 631900.55 & 51.6 & $\mathrm{c} 1$ & $2 \times 900$ & 0.158 & $\mathrm{AbL}$ & 17.2 & 13.4 & II \\
\hline
\end{tabular}

${ }^{a}$ Redshift taken from the NASA Extragalactic Database.

${ }^{b}$ Redshift from Schoenmakers et al. (1999).

aspects. Those radio galaxies with a difficult distinction between the FR I or FR II types are labelled as I/II.

We plot in Fig. 3 a histogram of the apparent $R$-band magnitude of the galaxies in the sample. The magnitude distribution is very extended, ranging from 8.2 up to magnitude 19.7 with a peak lying between the 15th and 16th magnitudes. The distribution presents a tail towards brighter objects induced by the poor calibration of photographic plates due to their non-linear behavior. At the other extreme the distribution is influenced by the limiting sensitivity of the DSS $\left(m_{R} \leq 20\right)$.

In Fig. 4 we represent the apparent de-reddened $R$-band magnitude as a function of redshift for the sample members, excepting the three quasar type objects in the sample. We have also added in this plot the radio galaxies (excepting quasars) from the sample constructed by Schoenmakers et al. (2001), who provide a photometry

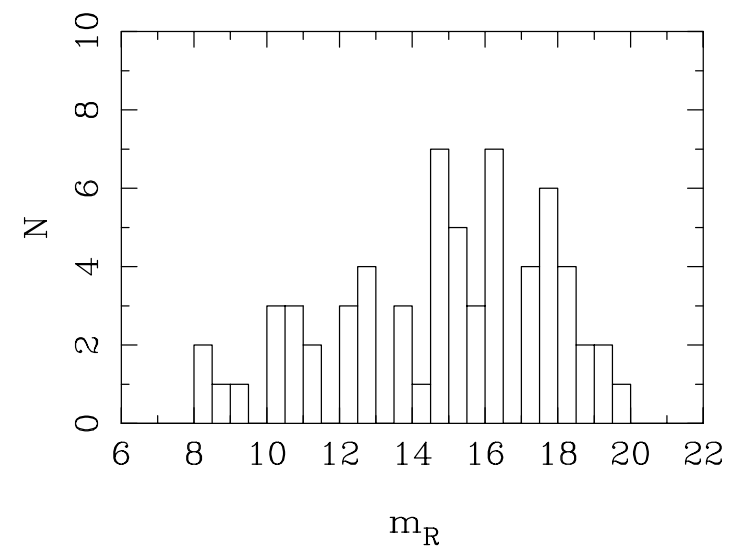

Fig. 3. Histogram of the apparent $R$-band magnitudes of the galaxies in our sample. The bin size is 0.5 magnitudes.

consistent with ours. We find a relation of the form (least squares fitting)

$m_{R}-A(R)=(8.83 \pm 0.35) \log z+(22.96 \pm 0.37)$ 
Table 2. continued.

\begin{tabular}{|c|c|c|c|c|c|c|c|c|c|c|}
\hline Name & $\begin{array}{c}\text { RA (J2000.0) } \\
\left(\begin{array}{lll}\left(\begin{array}{lll}\mathrm{h} & \mathrm{m} & \mathrm{s}\end{array}\right) \\
\end{array}\right.\end{array}$ & $\begin{array}{c}\text { Dec (J2000.0) } \\
\left({ }^{\circ},{ }^{\prime} \quad{ }^{\prime}\right)\end{array}$ & $\begin{array}{c}b \\
\left({ }^{\circ}\right) \\
\end{array}$ & Epoch & $\begin{array}{r}T_{\exp } \\
(\mathrm{s})\end{array}$ & $z$ & Notes & $\begin{array}{c}m_{R} \\
(\mathrm{mag})\end{array}$ & $\begin{array}{c}r_{\mathrm{e}} \\
(\mathrm{kpc})\end{array}$ & $\begin{array}{l}\text { Radio } \\
\text { Type }\end{array}$ \\
\hline J1504+689 & $\begin{array}{llll}15 & 04 & 12.781\end{array}$ & $6856 \quad 12.75$ & 43.9 & & & $0.318^{a}$ & SEL & 16.2 & - & II-QSS \\
\hline $\mathrm{J} 1523+636$ & 152345.900 & 633923.78 & 46.0 & & & $0.204^{a}$ & SEL & 16.0 & - & II \\
\hline $\mathrm{J} 1530+824$ & 153056.110 & $82 \quad 2721.02$ & 32.8 & & & $0.021^{a}$ & $? ?$ & 9.1 & - & I \\
\hline $\mathrm{J} 1536+843$ & 153657.335 & $8423 \quad 10.42$ & 31.3 & & & $0.201^{b}$ & WEL & 18.2 & - & II \\
\hline $\mathrm{J} 1557+706$ & 155730.190 & 704120.79 & 39.3 & & & $0.026^{a}$ & $\mathrm{AbL}$ & 8.2 & - & I \\
\hline $\mathrm{J} 1632+825$ & 163231.630 & $8232 \quad 16.28$ & 31.2 & & & $0.023^{a}$ & SEL & 8.4 & - & I \\
\hline $\mathrm{J} 1650+815$ & 165058.686 & 813428.11 & 31.1 & & & $0.038^{a}$ & $? ?$ & 11.3 & - & I \\
\hline $\mathrm{J} 1732+714$ & 173233.001 & 712410.50 & 31.9 & & & $0.059^{a}$ & $? ?$ & 12.0 & - & I \\
\hline $\mathrm{J} 1733+707$ & 173312.525 & 704630.36 & 31.9 & & & $0.041^{a}$ & $? ?$ & 11.4 & - & I \\
\hline $\mathrm{J} 1743+712$ & 174317.681 & $71 \quad 1253.98$ & 31.0 & & & $(0.25)$ & - & 17.8 & - & II \\
\hline $\mathrm{J} 1745+712$ & 174543.573 & 711548.55 & 30.8 & & & $0.216^{a}$ & SEL & 18.4 & - & II \\
\hline $\mathrm{J} 1751+680$ & 175119.629 & 680443.05 & 30.6 & b4 & $2 \times 900$ & 0.079 & $\mathrm{AbL}$ & 12.4 & 43.6 & I \\
\hline $\mathrm{J} 1754+626$ & 175450.310 & 623841.96 & 30.3 & & & $0.028^{a}$ & WEL & 10.3 & - & I \\
\hline $\mathrm{J} 1800+717$ & 180042.622 & 714441.99 & 29.6 & b4 & & $-\quad(\geq 0.40)$ & - & $\geq 19.5$ & - & II \\
\hline $\mathrm{J} 1835+665$ & 183507.338 & 663500.02 & 26.3 & b3 & $2 \times 1200$ & $0.354 ?(0.34)$ & $\mathrm{AbL}$ & 19.0 & 14.6 & II \\
\hline $\mathrm{J} 1835+620$ & 183510.405 & $\begin{array}{llll}62 & 04 & 07.42\end{array}$ & 25.6 & b1 & $2 \times 1200$ & 0.518 & SEL & - & - & II \\
\hline $\mathrm{J} 1844+653$ & 184407.443 & $\begin{array}{lll}65 & 2203.07\end{array}$ & 25.2 & b1 & $2 \times 1200$ & 0.197 & $\mathrm{AbL}$ & 17.1 & - & II \\
\hline $\mathrm{J} 1845+818$ & $1845 \quad 15.836$ & 814930.98 & 27.0 & b1 & $2 \times 900$ & 0.119 & $\mathrm{AbL}$ & 14.3 & 30.6 & II \\
\hline $\mathrm{J} 1847+707$ & 184734.912 & 704400.64 & 25.8 & a1 & $500+900$ & 0.043 & $\mathrm{AbL}$ & 10.6 & 13.4 & I \\
\hline $\mathrm{J} 1850+645$ & 185045.871 & 643024.68 & 24.4 & a1 & $2 \times 900$ & 0.080 & $\mathrm{AbL}$ & 12.6 & 21.6 & I \\
\hline $\mathrm{J} 1853+800$ & 185352.077 & 800250.46 & 26.6 & & & $0.214^{a}$ & $? ?$ & 17.8 & - & II \\
\hline $\mathrm{J} 1918+742$ & 191834.885 & 741505.05 & 24.2 & b2 & $2 \times 1200$ & 0.194 & WEL & 17.5 & 8.2 & II \\
\hline $\mathrm{J} 1951+706$ & 195140.825 & 703739.99 & 20.7 & b3 & $2 \times 1200$ & 0.550 & SEL & - & - & II \\
\hline $\mathrm{J} 2016+608$ & 201618.630 & 605357.49 & 14.0 & $\mathrm{c} 1$ & $2 \times 900$ & 0.121 & SEL & 18.2 & 10.6 & II \\
\hline $\mathrm{J} 2035+680$ & 203516.549 & $\begin{array}{lll}68 & 05 & 41.60\end{array}$ & 16.1 & b2 & $2 \times 1200$ & 0.133 & WEL & 18.0 & 9.9 & I \\
\hline $\mathrm{J} 2042+751$ & $2042 \quad 37.180$ & $\begin{array}{lll}75 & 08 & 02.52\end{array}$ & 19.5 & & & $0.104^{a}$ & SEL & 14.7 & - & II-QSS \\
\hline $\mathrm{J} 2059+627$ & 205909.560 & $62 \quad 4744.11$ & 11.0 & $\mathrm{c} 2$ & $2 \times 1200$ & 0.267 & SEL & - & - & II? \\
\hline $\mathrm{J} 2103+649$ & 210313.868 & 645655.26 & 12.0 & $\mathrm{c} 2$ & $2 \times 1200$ & 0.215 & $\mathrm{AbL}$ & - & - & II \\
\hline $\mathrm{J} 2111+630$ & 211120.268 & 630006.17 & 10.1 & $\mathrm{c} 1$ & $2 \times 900$ & - & - & - & - & II \\
\hline $\mathrm{J} 2114+820$ & $21 \quad 1401.179$ & 820448.28 & 22.3 & a1 & $2 \times 900$ & 0.085 & SEL & 13.5 & - & I \\
\hline $\mathrm{J} 2128+603$ & 212802.634 & 602107.96 & 6.8 & b2 & $2 \times 1200$ & $0.072 ?$ & $\mathrm{AbL}$ & - & - & II \\
\hline $\mathrm{J} 2138+831$ & 213842.266 & $\begin{array}{llll}83 & 06 & 49.21\end{array}$ & 22.4 & b2 & $2 \times 900$ & 0.135 & WEL & 14.5 & 36.9 & $\mathrm{I} / \mathrm{II}$ \\
\hline $\mathrm{J} 2145+819$ & 214529.887 & 815454.22 & 21.4 & & & $0.146^{b}$ & SEL & 17.1 & - & II \\
\hline $\mathrm{J} 2157+664$ & 215702.572 & $\begin{array}{lll}66 & 26 & 10.24\end{array}$ & 9.2 & b3 & $2 \times 900$ & $0.057 ?$ & $\mathrm{AbL}$ & - & - & $\mathrm{I} / \mathrm{II}$ \\
\hline $\mathrm{J} 2204+783$ & 220409.225 & $7822 \quad 46.92$ & 18.2 & a1 & $2 \times 900$ & 0.115 & $\mathrm{AbL}$ & 15.1 & 15.5 & II \\
\hline $\mathrm{J} 2209+727$ & 220933.780 & 724558.36 & 13.6 & $\mathrm{c} 2$ & $2 \times 1200$ & 0.201 & WEL & - & - & II \\
\hline $\mathrm{J} 2242+622$ & $2242 \quad 32.133$ & $62 \quad 12 \quad 17.53$ & 3.0 & c1 & $2 \times 1200$ & $0.188 ?$ & $\mathrm{AbL}$ & - & - & II \\
\hline $\mathrm{J} 2247+633$ & 224729.714 & 632115.55 & 3.7 & $\mathrm{c} 2$ & & - & - & - & - & I \\
\hline $\mathrm{J} 2250+729$ & 225043.621 & $7256 \quad 16.19$ & 12.1 & c1 & & - & - & - & - & II \\
\hline $\mathrm{J} 2255+645$ & 225529.943 & 643006.86 & 4.4 & $\mathrm{c} 2$ & & - & - & - & - & II \\
\hline $\mathrm{J} 2307+640$ & 230758.533 & 640139.22 & 3.4 & $\mathrm{c} 2$ & & - & - & - & - & II \\
\hline $\mathrm{J} 2340+621$ & 234056.435 & 621045.09 & 0.4 & b3 & & - & - & - & - & $\mathrm{I}$ \\
\hline
\end{tabular}

${ }^{a}$ Redshift taken from the NASA Extragalactic Database.

${ }^{b}$ Redshift from Schoenmakers (1999).

with a correlation coefficient $r=0.942$ and $\mathrm{rms}=$ 1.01 mag. It allows us to estimate, with a typical error of $\sim 30 \%$, the redshift of those galaxies with known magnitudes for which an spectroscopic determination was not possible (values shown in parenthesis in Table 2). For those galaxies with an uncertain spectroscopic redshift, a "photometric" estimation is also provided when possible (shown in parenthesis).

The fact that such a tight correlation between apparent magnitude and redshift exists is an indication that the host galaxies of the sample members have quite similar properties and that a possible orientation dependent beaming of the core emission does not play a significant role as expected for AGNs with their main axis oriented close to the plane of the sky, contrary to favorably oriented objects like blazars.

\section{Conclusions}

We have made optical observations pointing at the core position of 57 radio galaxies from our sample and in all cases, except one, we have detected the optical galaxy responsible of the radio emission. Even the galaxy $\mathrm{J} 2340+621$, at the very low galactic latitude of $+0.4^{\circ}$ has been tentatively detected. The observed galaxies have profiles consistent with being elliptical. We took spectra of 48 galaxies, of which the redshift could be determined with high confidence for 40 of them and with uncertainty for 6 galaxies. For only 2 galaxies, J0828+632 and J2111+630, we were unable to determine the redshift. However, a "photometric" redshift can be estimated from a correlation derived between apparent magnitudes and spectroscopic redshift of the sample members. 


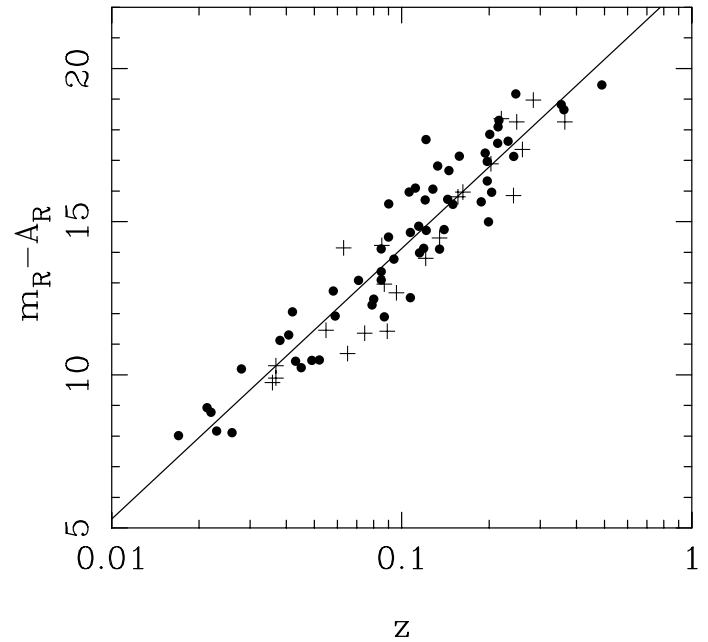

Fig. 4. De-reddened apparent $R$-band magnitudes of the members of our sample (dots) and Schoenmakers' et al. sample (crosses), plotted against their redshifts. The solid line represents a linear fit to the data.

Regarding the properties of the optical spectrum, $36 \%$ of the galaxies present spectra with prominent emission lines, $19 \%$ of the galaxies show weak emission lines with respect to the continuum emission, and $45 \%$ of the galaxies have spectra characterized by stellar absorption lines only.

The fact that our sample is designed to select radio galaxies with their main axes oriented on the plane of the sky is consistent with the spectroscopic results, since only 3 galaxies (J0342+636, J0750+656 and J2114+820) out of 48 observed present broad emission lines, as expected for objects oriented towards the observer according to current unification schemes (e.g. Antonucci 1993).

In a forthcoming paper of this series, we will discuss the possible relations between the different parameters derived from the radio and optical observations presented in this paper and in Paper I, and the possible dependencies of these parameters with the type of radio structure.
Acknowledgements. We thank F. Govoni and I. GonzálezSerrano for helpful discussion. This research is supported in part by the Spanish DGICYT (PB97-1164). LF and GG acknowledges the Italian Ministry for University and Research (MURST) for financial support under grant Cofin98-02-32. The National Radio Astronomy Observatory is a facility of the National Science Foundation operated under cooperative agreement by Associated Universities, Inc. This research has made use of the NASA/IPAC Extragalactic Database (NED) which is operated by the Jet Propulsion Laboratory, California Institute of Technology, under contract with the National Aeronautics and Space Administration, and of the Aladin interactive sky atlas, CDS, Strasbourg, France.

\section{References}

Antonucci, R. 1993, ARA\&A, 31, 473

Condon, J. J., Cotton, W. D., Greisen, E. W., et al. 1998, AJ, 115, 1693

Doggett, J., Postman, M., Lasker, B. M., \& Meakes, M. 1996, in Astronomical Data Analysis Software and Systems V, ed. H. George, J. Barnes, \& J. Barnes, ASP Conf. Ser., 101,159

Fanaroff, B. L., \& Riley, J. M. 1974, MNRAS, 167, 31

Lara, L., Cotton, W. D., Feretti, L., et al. 2001, A\&A, 370, 409, Paper I

Lara, L., Márquez, I., Cotton, W. D., et al. 1999, NewAR, 43, 643

Machalski, J., Jamrozy, M., \& Zola, S. 2001, A\&A, 371, 445

Monet, D., Bird, A., Canzian, B., et al. 1998, U.S. Naval Observatory Flagstaff Station and Universities Space Research Association stationed at USNOFS

Schlegel, D. J., Finkbeiner, D. P., \& Davis, M. 1998, ApJ, 500, 525

Schoenmakers, A. P. 1999, Ph.D. Thesis, University of Utrecht Schoenmakers, A. P., Mack, K.-H., de Bruyn, A. G., et al. 2000, A\&AS, 146, 293

Schoenmakers, A. P., de Bruyn, A. G., Röttgering, H. J. A., \& van der Laan, H. 2001, A\&A, 374, 861 